\title{
Approximate Solution of Hyper-Sensitive Optimal Control Problems Using Finite-Time Lyapunov Analysis
}

\author{
Erkut Aykutlug and Kenneth D. Mease
}

\begin{abstract}
Solving optimal control problems by an indirect method is often abandoned in favor of a direct method due to hyper-sensitivity with respect to unknown boundary conditions for the Hamiltonian boundary-value problem that represents the first-order necessary conditions. Yet the hyper-sensitivity may imply a manifold structure for the flow in the Hamiltonian phase space, structure that provides insight regarding the optimal solutions and suggests a solution approximation strategy that avoids the hyper-sensitivity. This paper concerns the development of a solution approximation method based on finite-time Lyapunov exponents and vectors. The focus is on determining the unknown boundary conditions such that the solution end points lie on certain invariant manifolds. Using kinematic eigenvalues, a systematic approach to determine the appropriate finite-time for the Lyapunov analysis is presented. A simple example is used to illustrate the approximation method and its implementation.
\end{abstract}

\section{INTRODUCTION}

Numerical methods for solving optimal control problems (OCPs) can be divided into two main categories, direct and indirect. Indirect methods solve the associated Hamiltonian boundary value problem (HBVP) constructed from the firstorder necessary conditions. A survey of direct and indirect methods, noting their advantages and disadvantages, is given in [6].

An OCP is called hyper-sensitive if the final time is long relative to some of the contraction and expansion rates of the Hamiltonian system associated with it [15], [16]. The solution to a hyper-sensitive problem has a "take-off", "cruise", and "landing" structure analogous to the optimal flight of an aircraft between distant locations. The "cruise" segment is primarily determined by the cost function and the state dynamics, whereas the "take-off" and "landing" segments are determined by the boundary conditions and the goal of connecting these to the "cruise" segment. As the final time increases so does the time spent in the cruise segment which shadows a slow reduced-order manifold. When the final time is long, the sensitivity of the final state to the unknown initial conditions makes the HBVP ill-conditioned. The ill-conditioning can be removed by approximating the solution with a concatenation of boundary-layer solutions (take-off/landing segments) with a solution segment on the slow manifold (cruise segment). The completely hypersensitive case is a degenerate case for which, rather than a slow manifold, there is an equilibrium point, and the "cruise

This research was supported in part by NSF Grant CMS-0010085

E. Aykutluğ, Mechanical and Aerospace Engineering, University of California, Irvine, CA 92697, eaykutlu@uci.edu

K. D. Mease, Mechanical and Aerospace Engineering, University of California, Irvine, CA 92697, kdmease@uci.edu segment" is near-equilibrium motion. The more general case in which the cruise segment shadows a trajectory on the slow manifold is called partially hyper-sensitive. In this paper, we continue the development of a solution strategy for completely hyper-sensitive problems that is extendable to the partially hyper-sensitive case.

Solution approximation for completely hyper-sensitive optimal control problems, based on the geometric structure of the associated Hamiltonian dynamics, has been addressed in [3], [15]. The solution to the HBVP is such that the solution in the initial boundary-layer is approximated by a trajectory on the stable manifold of the equilibrium point, the solution in the final boundary-layer is approximated by a trajectory on the unstable manifold of the equilibrium, and the boundary-layer solutions are approximately matched near the equilibrium point. The focus of the present paper is on determining the unknown boundary conditions such that the solution end points lie on the appropriate invariant manifolds to sufficient accuracy.

Rather than use information related to the equilibrium point which would not be available in the partially hypersensitive case, our approach uses a dichotomic basis for the phase space tangent bundle to define conditions satisfied by points on the stable and unstable manifolds of an equilibrium point. Dichotomic transformations have been used to solve boundary-value problems for ordinary differential equations [4] and to solve fixed end-point optimal control problems [2], [20]. Chow used dichotomic transformations to solve nonlinear HBVPs with linear boundary-layer dynamics [8]. For nonlinear HBVPs, a dichotomic basis has been approximated using eigenvalues and eigenvectors (eigen-analysis) [15], [16] and finite-time Lyapunov exponents and vectors [18]. The latter information can provide greater accuracy and is more generally applicable [13], [14]. Finite-time Lyapunov exponents, and in some cases vectors, have also been used to analyze fluids [9], [11], [17] and atmospheric circulation, e.g. [7]. A primary contribution of the present paper, relevant more generally to the use of finite-time Lyapunov exponents and vectors, is a systematic approach to determine the appropriate finite-time for Lyapunov analysis, using kinematic eigenvalues [4], [9], [13], [19].

\section{Optimal Control Problem and Associated HaMiltonian BOUNDARY-VALUe PROBLEM}

We consider the Lagrangian optimal control problem: determine the control function $u$ on the time interval $\left[0, t_{f}\right]$ 
that minimizes the cost function

$$
\mathbf{J}=\int_{0}^{t_{f}} L(x(t), u(t)) d t
$$

$$
\begin{array}{cc} 
& \dot{x}=f(x, u) \\
& x(0)=x_{0}, x\left(t_{f}\right)=x_{f}
\end{array}
$$

where we assume that the vector field, $f(x, u)$, and $L(x, u)$ are smooth in both $x$ and $u$, and that $t_{f}$ is given. The state vector $x(t) \in \mathbb{R}^{n}$ and the control $u(t) \in \mathbb{R}^{m}$. The first-order necessary conditions for optimality lead to the Hamiltonian boundary-value problem (HBVP):

$$
\begin{array}{crrr}
\dot{x} & = & \frac{\partial H^{*}}{\partial \lambda} \\
\dot{\lambda} & = & -\frac{\partial H^{*}}{\partial x} \\
x(0)=x_{0}, \text { and } & x\left(t_{f}\right)=x_{f},
\end{array}
$$

where $\lambda(t) \in \mathbb{R}^{n}$ is the costate vector and $H^{*}=\mathcal{L}\left(x, u^{*}\right)+$ $\lambda^{T} f\left(x, u^{*}\right)$ is the Hamiltonian evaluated at the optimal control $u^{*}(x(t), \lambda(t))=\arg \min H(x(t), \lambda(t), u(t))$. We assume $u^{*}$ is a smooth function of $x$ and $\lambda$. The augmented state $p=(x, \lambda)$ is a point in the phase space $\mathbb{R}^{2 n}$. In terms of $p$, we write the Hamiltonian dynamics in (2) as

$$
\dot{p}=h(p)
$$

where $h(p)$ is the Hamiltonian vector field. The corresponding Hamiltonian flow is expressed as $\phi\left(t, p_{o}\right)$ where $p_{o}$ is the initial phase and $\phi\left(0, p_{o}\right)=p_{o}$. A Hamiltonian vector field is divergence free; in other words, the flow preserves volume. Therefore for every direction in which the Hamiltonian flow contracts, there is a direction in which it expands.

\section{Approximate Solution Strategy for A COMPletely HyPER-SENSITIVE Problem}

For clarity, we present the approximate solution strategy for a completely hyper-sensitive optimal control problem via a simple transparent example. Consider the optimal control problem [12] to determine the scalar control $u^{*}$ and corresponding trajectory for the scalar state $x^{*}$ that minimize the cost

$$
\mathbf{J}=\frac{1}{2} \int_{0}^{t_{f}} u^{2} d t
$$

subject to the dynamic constraint

$$
\dot{x}=\sin x+u
$$

for a given final time $t_{f}$ and boundary conditions $x(0)=1$ and $x\left(t_{f}\right)=-1$. The first-order necessary conditions lead to the following Hamiltonian boundary value problem (HBVP)

$$
\begin{array}{cll}
\dot{x} & = & \sin x-\lambda \\
\dot{\lambda} & = & -\lambda \cos x \\
x(0)=1, & & x\left(t_{f}\right)=-1
\end{array}
$$

Numerical solutions for the OCP were obtained using the optimization program GESOP ${ }^{\complement}$ for different final times; see Fig. 1. GESOP has several options, of these we used the direct multiple shooting method. The optimal trajectory and
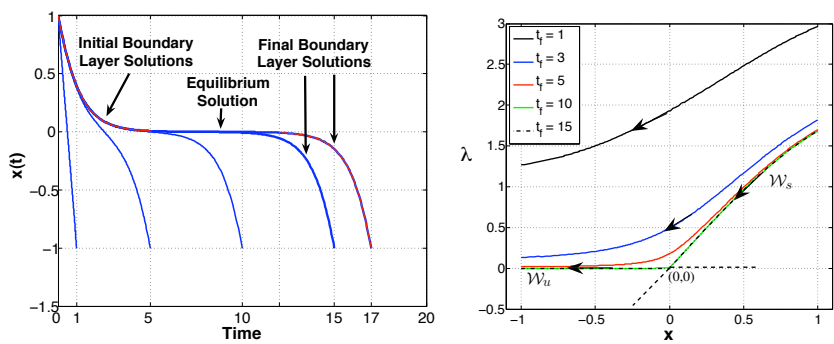

Fig. 1. Optimal solutions from GESOP ${ }^{(C)}$ for different final times, $t_{f}=$ $1,5,10,15$ and 17 , where $x(0)=1$ and $x\left(t_{f}\right)=-1$, in the time domain (left) and in phase space (right).

control are determined. Using the necessary condition, $u^{*}=$ $-\lambda$, the solutions are plotted in the $(x, \lambda)$ phase plane as well as in the time domain in Fig. 1. As the final time gets longer, the solution trajectories shadow more closely a branch of the stable, $\mathcal{W}^{s}$, and then a branch of the unstable, $\mathcal{W}^{u}$, invariant manifolds of the equilibrium point $p_{e q}$ at $(0,0)$. As the final time increases to $t_{f}=17$ the optimal solution spends more and more time near the equilibrium point. For larger times, it was increasingly difficult to obtain the solution. In contrast, as $t_{f}$ increases, the following approximate solution becomes more and more accurate and no harder to obtain.

For sufficiently large final times, the optimal strategy can be viewed in phase space as getting on $\mathcal{W}^{s}\left(p_{e q}\right)$ where $x(0)=1$ and steering along it to the equilibrium point, waiting at the equilibrium point an appropriate length of time, and then getting on $\mathcal{W}^{u}\left(p_{e q}\right)$ and steering along it until $x\left(t_{f}\right)=-1$ is reached. Consistent with this viewpoint, the solution to a completely hyper-sensitive OCP, $p^{*}(t)$, can be approximated by the composite function

$$
\hat{p}(t)= \begin{cases}\hat{p}_{s}(t) & 0 \leq t \leq t_{i b l} \\ p_{e q} & t_{i b l}<t \leq t_{f b l} \\ \hat{p}_{u}(t) & t_{f b l} \leq t \leq t_{f}\end{cases}
$$

where $\hat{p}_{s}(t)$ is the approximate initial boundary-layer solution for $t \in\left[0, t_{i b l}\right]$ with the initial condition on the stable manifold, i.e., $\hat{p}_{s}(0)=\left(x_{0}, \lambda_{0}\right) \in \mathcal{W}^{s}\left(p_{e q}\right) ; p_{e q}$ is the equilibrium solution which approximates the slow "cruise" segment; and $\hat{p}_{u}(t)$ is the approximate final boundary-layer solution for $t \in\left[t_{f b l}, t_{f}\right]$ with the final condition on the unstable manifold, i.e., $\hat{p}_{u}\left(t_{f}\right)=\left(x_{t_{f}}, \lambda_{t_{f}}\right) \in \mathcal{W}^{u}\left(p_{e q}\right)$. The solutions in the boundary-layers can be constructed by integrating, in forward and backward time respectively, the Hamiltonian dynamics from initial and final phase points on the corresponding invariant manifolds that satisfy the boundary conditions. The composite approximate solution is obtained by concatenating the boundary-layer solutions with the equilibrium solution. The times, $t_{i b l}$ and $t_{f b l}$, defining the initial and final boundary-layer durations, are selected such that $\hat{p}_{s}$ and $\hat{p}_{u}$ reach the equilibrium point up to a specified accuracy in forward and backward time respectively.

The primary challenge in developing this approach is to determine the unknown boundary conditions such that the initial and final phase points are sufficiently close to $\mathcal{W}^{s}\left(p_{e q}\right)$ and $\mathcal{W}^{u}\left(p_{e q}\right)$ respectively. The choice to base our approach 
on finite-time Lyapunov exponents and vectors (FTLE/Vs), rather than to exploit the local structure near the equilibrium point, is driven by the goal of extending the approach to the partially hyper-sensitive case.

\section{Finite-time LyapUnOV ANALysis AND DICHOTOMIC BASIS}

In [14] it was shown that finite-time Lyapunov analysis (FTLA) can be used to diagnose multiple timescale behavior in dynamical system models. In the present context, the goal of FTLA is to determine if the nonlinear Hamiltonian system, $\dot{p}=h(p)$, has a dichotomy in the phase space region of interest, and if so, to find a dichotomic basis for the tangent space, $T_{p} \mathbb{R}^{2 n}$, at each point $p$, with which to represent the splitting $T_{p} \mathbb{R}^{2 n}(p)=E^{s}(p) \oplus E^{u}(p)$, where all the vectors in the stable subspace $E^{s}(p)$ contract exponentially fast in forward time and all the vectors in the unstable subspace $E^{u}(p)$ contract exponentially fast in backward time, under the linearized Hamiltonian flow. In the asymptotic theory of hyperbolic systems, the stable and unstable subspaces are invariant subspaces, however, when defined in terms of FTLE/Vs, they only approximate invariant subspaces.

A tangent vector evolves according to the linearized dynamics, $\dot{v}=D h(p) v$, where $D h=\partial h / \partial p$. A vector $v \in T_{p} \mathbb{R}^{2 n}$, propagated $t$ units of time along the trajectory, $\phi(t, p)$, evolves into a vector $v(t)=\Phi(t, p) v$ where $v(t) \in$ $T_{\phi(t, p)} \mathbb{R}^{2 n}$ and $\Phi(t, p)$, with $\Phi(0, p)=I$, is the state transition matrix of the linearized dynamics along the trajectory. A multiplier, $\sigma$, which is the ratio of the norms of the final vector and the initial one, $\sigma(T, p, v)=\|v(T)\| /\|v\|$, characterizes the net expansion (resp. contraction) rate if $\sigma(T, p, v)>1$ (resp. $\sigma(T, p, v)<1$ ) over the time interval $[0, T]$. Here we take $\|\cdot\|$ to be the Euclidean norm. Finitetime Lyapunov exponents (FTLEs) in forward and backward time are defined as follows ${ }^{1}$.

$$
\begin{aligned}
& \mu^{+}(T, p, v)=\quad \frac{1}{T} \log \sigma^{+}(T, p, v)=\frac{1}{T} \ln \frac{\|\Phi(T, p) v\|}{\|v\|} \\
& \mu^{-}(T, p, v)=\quad \frac{1}{T} \log \sigma^{-}(T, p, v)=\frac{1}{T} \ln \frac{\|\Phi(-T, p) v\|}{\|v\|}
\end{aligned}
$$

where $p$ is the base point in phase space and $v$ is a vector in tangent space at $p$, i.e, $v \in T_{p} \mathbb{R}^{2 n}$. If $v=0$, both $\mu^{+}(T, p, v)$ and $\mu^{-}(T, p, v)$ are defined to be $-\infty$. The multiplier corresponding to an FTLE can be interpreted as an average exponential rate of a tangent vector, i.e., $\sigma(T, p, v)=$ $\exp (\mu(T, p, v) T)$, over the averaging time $T$.

A set of basis vectors, $l_{i}^{+}(T, p), i=1, \ldots, 2 n$, for $T_{p} \mathbb{R}^{2 n}$, is called normal, if the sum, $\sum_{i=1}^{2 n} \mu_{i}^{+}(T, p)$, where $\mu_{i}$ is the FTLE associated with $l_{i}$, is smallest relative to the sum for any other basis. One way to obtain such a set of vectors, referred to as finite-time Lyapunov vectors (FTLVs), is by computing a singular value decomposition (SVD) of the state transition matrix $\Phi(T, p)$. Let the SVD be given by $\Phi(T, p)=N^{+}(T, p) \Sigma^{+}(T, p) L^{+}(T, p)^{T}$, where the matrix $\Sigma^{+}(T, p)=\operatorname{diagonal}\left(\sigma_{1}^{+}(T, p), \ldots, \sigma_{2 n}^{+}(T, p)\right)$ contains the singular values in an ascending order, i.e.,

\footnotetext{
${ }^{1}$ Throughout this paper the superscripts ${ }^{+}$and ${ }^{-}$will denote forward and backward time respectively.
}

$\sigma_{1}^{+}(T, p) \leq \ldots \leq \sigma_{2 n}^{+}(T, p)$. The FTLEs are evaluated by $\mu_{i}^{+}(T, p)=\frac{1}{T} \log \sigma_{i}^{+}(T, p)$. Then the column vectors of $L^{+}(T, p), l_{i}^{+}(T, p), i=1, \ldots, 2 n$, constitute the normal basis for $T_{p} \mathbb{R}^{2 n}$. Due to the SVD properties, the basis vectors are also mutually orthogonal and unit length. The geometric view is that when a unit $2 n-$ sphere in $T_{p} \mathbb{R}^{2 n}$ is propagated along the trajectory forward in time, it becomes an ellipsoid in $T_{\phi(T, p)} \mathbb{R}^{2 n}$. The principal axes are aligned in the direction of the vectors $n_{i}^{+}, i=1, \ldots, 2 n$, the column vectors of the $N^{+}$matrix, with corresponding lengths $\exp \left(\mu_{i}^{+} T\right)$. We can define the backward FTLE/Vs in the same way. The backward exponents are assumed to be in descending order, $\mu_{1}^{-} \geq \ldots \geq \mu_{2 n}^{-}$. Analogous to the asymptotic theory [5], we construct finite-time subspaces

$$
\begin{aligned}
\mathcal{L}_{i}^{+}(T, p) & =\operatorname{span}\left\{l_{1}^{+}(T, p), \ldots, l_{i}^{+}(T, p)\right\} \\
\mathcal{L}_{i}^{-}(T, p) & =\operatorname{span}\left\{l_{i}^{-}(T, p), \ldots, l_{2 n}^{-}(T, p)\right\}
\end{aligned}
$$

Hamiltonian systems have the property that the FTLEs are always symmetrically distributed on the real line with respect to the origin. We say that the Hamiltonian system $\dot{p}=h(p)$ has a stable/unstable dichotomy on a neighborhood of the optimal trajectory $p^{*}$, denoted $\mathcal{N}\left(p^{*}\right)$, if there exist positive constants $\underline{\mu}, \underline{T}$, and $\bar{T}$ with $\underline{T}<\bar{T}$ such that $\mu_{n+1}^{+}(T, p) \geq \underline{\mu}$ and $\mu_{n}^{-}(\bar{T}, p) \geq \underline{\mu}$ for $T \in[\underline{T}, \bar{T}]$ for all $p \in \mathcal{N}\left(p^{*}\right)$. If there is a dichotomy and the time constant $\underline{\mu}^{-1}$ is a small fraction of both $t_{f}$ and $\bar{T}$, then the optimal control problem and the associated HBVP are completely hyper-sensitive. If there exists a dichotomy, then a stable/unstable splitting can be defined using $E^{s}(p)=\mathcal{L}_{n}^{+}(\bar{T}, p)$ and $E^{u}(p)=\mathcal{L}_{n+1}^{-}(\bar{T}, p)$ for any $p \in \mathcal{N}\left(p^{*}\right)$. Equivalently, we can determine a dichotomic basis using $l_{1}^{+}, \ldots, l_{n}^{+}$as the columns of the matrix $D^{s}(p)$ and $l_{n+1}^{-}, \ldots, l_{2 n}^{-}$as the columns of the matrix $D^{u}(p)$. Further, the vector field, $h(p)$, can be expressed in terms of the dichotomic basis

$$
\dot{p}=h(p)=h_{s}(p)+h_{u}(p)=D^{s}(p) w_{s}(p)+D^{u}(p) w_{u}(p)
$$

where $h_{s}(p)$, and $h_{u}(p)$ are the stable and the unstable components of $h$ at $p$. A point $p \in \mathcal{W}^{s}\left(p_{e q}\right)$ (resp. $p \in \mathcal{W}^{u}$ ) satisfies the partial equilibrium condition $w_{u}(p)=0$ (resp. $\left.w_{s}(p)=0\right)$. The partial equilibrium conditions $w_{u}(p)=0$ at $t=0$ and $w_{s}(p)=0$ at $t=t_{f}$ can be used to determine the unknown boundary conditions such that the initial and final phase points lie on the appropriate invariant manifolds within some level of accuracy [18].

A related method, referred to as maxFTLE, was developed in [9], [11], [17], to identify invariant manifolds of interest (Lagrangian coherent structures), from the maximal level contours of the maximum FTLE field, called the ridges. One could use the maxFTLE method to get an estimate of the location of $\mathcal{W}^{s}$. Phase points closer to the invariant manifolds will have the largest maximum FTLE for longer times $T$ than points farther away from the invariant manifolds as will be shown in Fig. 4 to be described later. Therefore the ridges indicate the invariant manifolds. Both methods maxFTLE and FTLA require the selection of the averaging time $T$. One of the contributions of the paper regards this 
issue. We show how kinematic eigenvalues provide guiding information. Also with the primary tools in FTLA being the FTLVs and the understanding of their convergence rate from our previous work [14], we can further clarify how to choose $T$.

A kinematic eigenvalue (KE) quantifies the instantaneous exponential rate of change of a tangent vector under the linearized flow [9], [13], [19]. The kinematic eigenvalue of a tangent vector, $v(t)$, evolving according to the linearized Hamiltonian dynamics, $\dot{v}=D h(p) v$, is evaluated as follows. Define a unit vector $e(t)=v(t) /\|v(t)\|$, where $v(t) \in$ $T_{p} \mathbb{R}^{2 n}$. The differential equation governing the dynamics of $e(t)$ is $\dot{e}=[D h-\rho(t) \cdot I] e$ where $\rho(t)=\frac{1}{2} e^{T}\left(D h^{T}+D h\right) e$ is the $\mathrm{KE}$ associated with the tangent vector $e$ and defines the local expansion/contraction rate in the $e$ direction. When the normalized tangent vectors, $e_{i}(t), i=1, \ldots, 2 n$ are initialized with the $l_{i}(T, x)$ vectors, the time average of $\rho_{i}(t)$, $\bar{\rho}_{i}(t):=\frac{1}{T} \int_{0}^{T} \rho_{i}(\tau) d \tau=\mu_{i}(T, p)$, the corresponding FTLE.

The instantaneous rate of change of the distance between neighboring points on the same trajectory can be monitored by computing the KE for the vector field, $h(p)$, given by

$$
\rho_{h}(p)=\frac{1}{2} h^{T}(p)\left(D h^{T}(p)+D h(p)\right) h(p)
$$

Near an equilibrium point, neighboring points on a trajectory that shadows a branch of the stable (resp. unstable) invariant manifold become closer to (resp. farther apart from) each other with time. On a trajectory shadowing a heteroclinic invariant manifold, $\rho_{h}$ will cross zero.

\section{Determining Boundary Conditions on Stable AND UNSTABLE MANIFOLdS USING FINITE-TIME LYAPUNOV ANALYSIS}

We use a method based on FTLA to identify the unknown boundary conditions such that the initial and final phase points will lie on the appropriate invariant manifolds. In particular, we describe how to determine the phase point on $\mathcal{W}^{s}$ that is also on the line $x=x_{0}$. The same approach can be applied to determine the final boundary condition on $\mathcal{W}^{u}$. FTLA starts with computing the finite-time Lyapunov exponents (FTLEs) over a set, $\mathcal{Y}=\left\{(x, \lambda) \in \mathbb{R}^{2 n} \mid x=\right.$ $\left.x_{0}, \lambda \in \mathbb{R}^{n}\right\}$ in the Hamiltonian phase space. All points in $\mathcal{Y}$ satisfy the initial boundary condition. Initially we assume we do not have any information on the optimal solution. We solve the HBVP for several shorter final times using GESOP $^{(}[1]$, which allows us to identify a subset of $\mathcal{Y}$, $\mathcal{P}=\left\{(x, \lambda) \in \mathbb{R}^{2 n} \mid x=x_{0}, \lambda \in \mathbb{R}^{n}\right\}$, expected to contain the optimal initial phase point $p_{0}=\left(x_{0}, \lambda_{0}\right)$ and to determine the equilibrium point $p_{e q}$ that the optimal solution stays near for large $t_{f}$. The FTLE/Vs are computed on a grid on $\mathcal{P}$. If $p$ lies on $\mathcal{W}^{s}\left(p_{e q}\right)$, then $h(p) \in E^{s}(p)$, where $E^{s}(p)$ is the stable subspace at $T_{p} \mathcal{P}$ as illustrated in Fig. 3. Then a point on $\mathcal{W}^{s}$ would satisfy

$$
<h(p), v>=0, \forall v \in\left(E^{s}\right)^{\perp}(p) .
$$

Using FTLA [14] we can get approximations of the invariant stable and the unstable spaces at point $p, \hat{E}^{s}(T, p)$, and

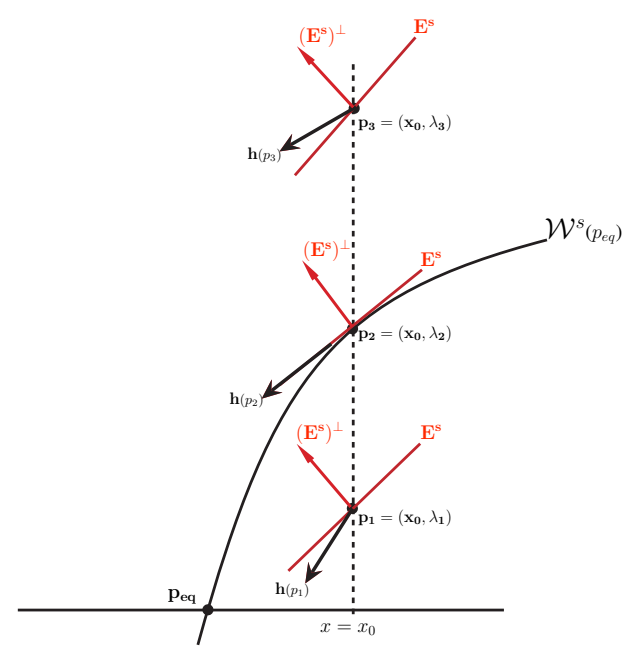

Fig. 3. Illustrating subspaces and orthogonality conditions.

$\hat{E}^{u}(T, p)$ respectively, in a neighborhood of $\mathcal{W}^{s}$. Starting from an initial guess $p$ we apply the orthogonality condition in (11) to get an estimate $\hat{p}$ of a point on $\mathcal{W}^{s}$. Then we re-initialize the FTLA method at $\hat{p}$ and repeat. It was demonstrated in [14] that FTLA can accurately identify points on $\mathcal{W}^{s}$.

Consider the HBVP given in (6). There are multiple equilibria at $p=(n \pi, 0), n \in \mathbb{Z}, p=(\pi / 2+2 n \pi, 1)$, $n \in \mathbb{Z}$ and $p=(-\pi / 2+2 n \pi,-1), n \in \mathbb{Z}$. The equilibria at $p=(n \pi, 0), n \in \mathbb{Z}$ are connected by heteroclinic invariant manifolds. The phase portrait emphasizing the connections and neighboring trajectories is shown in Fig. 2. The trajectories with initial conditions inside the loops are closed curves (periodic orbits), whereas the ones outside the loops are unbounded as $t \rightarrow \infty$ with $x \rightarrow-\infty$ if $\lambda>0$ and $x \rightarrow+\infty$ if $\lambda<0$.

Analyzing the linearized dynamics, the asymptotic Lyapunov exponents are zero almost everywhere except on the heteroclinic connections. On the other hand FTLA can reveal the manifold structure around an equilibrium point. Fig. 2 shows the 'lines of ambiguity' at $x=-\pi / 2$ and $x=\pi / 2$. At these values of $x$, the orthogonality condition (11) is satisfied for all values of $\lambda$ and cannot be used to identify the particular value of $\lambda$ that would place $p$ on the appropriate invariant manifold. For all other values of $x$ in this range, there are isolated solutions to the orthogonality condition corresponding to the desired manifolds.

A systematic approach to selecting the averaging time $T$ such that the FTLEs indicate the local nature of the flow to sufficient accuracy, for a flow with non-uniform kinematic eigenvalues is as follows. Fig. 2 shows trajectories that shadow the invariant manifolds of the equilibria. The $\mathrm{KE}$ for the vector field $h(p)$, given by

$$
\rho_{h}(t)=\frac{1}{2} h^{T}(p)\left((D h(p))^{T}+D h(p)\right) h(p)
$$

was computed along these trajectories. The red circles mark points where the $\rho_{h}$ is zero; it is positive on one side of such a point and negative on the other. The positive 


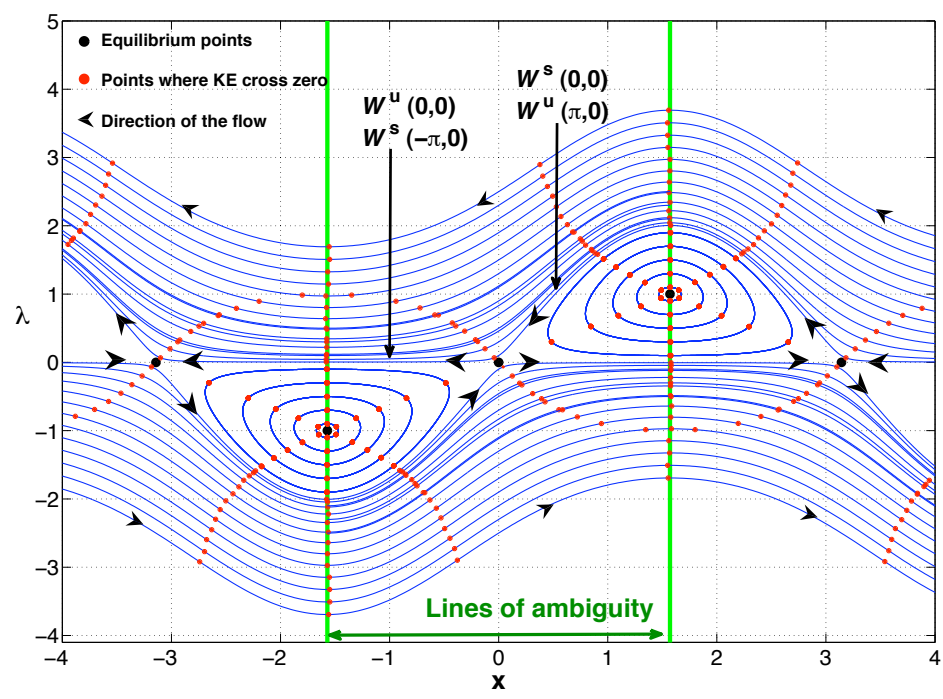

Fig. 2. Hamiltonian phase space of the system given in (6) showing stable and unstable manifolds of the equilibria, trajectories shadowing these manifolds, regions of uniformity (boundaries denoted by red circles), and lines of ambiguity.

sign indicates that neighboring points on the trajectory are separating with time, and negative sign indicates that they are getting closer to each other. Thus the red circles mark the boundaries separating regions of uniform (in sign) $\rho_{h}$. The time $T$ should be selected to average as long as possible over a uniform region. Fig. 4 shows for a grid of $\lambda(0)$ values, the trajectories that evolve in forward time from the corresponding initial phase points. The zero-crossing for $\rho_{h}$ are indicated, as are the times $T$ over which the FTLE/Vs can be computed to determine the stable subspace. Although some of the trajectories begin in a different uniform region, most of the time stated is spent in the uniform region where the attraction to the equilibrium point is sensed.

It has been shown in [10] that the FTLVs converge to their limits exponentially as the averaging time increases, so in this regard we want to averaging time to be as long as possible. On the other hand, we want to compute the FTLE/Vs along a trajectory only as long as the KEs are uniform, meaning here simply that they do not change sign, which determines the how long the averaging time can be.

\section{Approximate Solution of Lam's Problem}

Consider the HBVP given in (6) for final time $t_{f}=15$ with boundary conditions $x(0)=1, x\left(t_{f}\right)=-1$. Based on numerical solutions for shorter times, the initial costate $\lambda(0)$ is expected to be in the interval $[1,3]$. Therefore we initialize the FTLA method by computing the FTLEs on the set $\mathcal{P}=\left\{(x, \lambda) \in \mathbb{R}^{2} \mid x=1, \lambda \in[1,3]\right\}$. We have chosen a grid of points on $\mathcal{P}$ such that the costate values are equally spaced with increments of 0.05 .

To determine the point on the stable manifold at $x=1$, we need to find an approximation to the stable subspace at $x_{0}$, i.e., $l_{1}^{+}\left(T, p_{0}\right)$; therefore only forward integration is necessary. (Similarly to identify a point on the unstable manifold at $x_{f}=-1$, we need to find an approximation to the unstable subspace at $x_{f}$, i.e., $l_{2}^{-}$.)
Let us denote the initial phase point by $\phi\left(0, p_{0}^{(0)}\right):=$ $\left(x_{0}, \lambda_{0}^{(0)}\right)$, the $0^{\text {th }}$ iteration at $x_{0}$. We apply the FTLA method iteratively to get a point on the stable manifold. After each iteration the time interval over which the KE is uniform increases. Once the difference between the last two approximations to the point on $\mathcal{W}^{s}$ is less than a specified tolerance we take the last iteration, say it is the $k^{t h}$, value as the approximation of a point on $\mathcal{W}^{s}, \phi\left(0, p_{0}^{(k)}\right) . \mathcal{W}^{s}$ can be approximately constructed by integrating the system dynamics forward in time starting from this initial condition. FTLA can only compute a point that is approximately on $\mathcal{W}^{s}$ which means the trajectory will deviate from $\mathcal{W}^{s}$. Therefore we integrate the system dynamics for $t=T_{\text {reinit }}$, where $T_{\text {reinit }}$ is the time interval selected for re-initialization, forward in time from $\phi\left(0, p_{0}^{(n)}\right)$ to $\phi\left(T_{\text {reinit }}, p_{0}^{(n)}\right)$, and perform another FTLA iteration at $\phi\left(T_{\text {reinit }}, p_{0}^{(n)}\right)$ to pull the trajectory point back to $\mathcal{W}^{s}$. We continue this process until we reach the equilibrium point up to the desired accuracy. The optimal solution constructed in this manner for the system in (6) is shown in Fig. 5. The error between the re-initializations is largest at the beginning and can be decreased by using smaller $T_{\text {reinit }}$ values. The largest error is 0.14 when $T_{\text {reinit }}=0.2$ and 0.08 when $T_{\text {reinit }}=$ 0.1 . Comparison with the solution computed direct multiple shooting in GESOP shows that the maximum error is less than $1.2 \times 10^{-3}$ and decreases as the initial and final boundary layer solutions approach the equilibrium. The error increases around the equilibrium due to the discontinuity of the composite solution. The error at the equilibrium can be deceased by connecting the boundary-layer solutions with a continuous function.

\section{CONCLUSIONS}

A method to solve completely hyper-sensitive optimal control problems has been described. Finite-time Lyapunov analysis (FTLA) was used to construct an approximate 


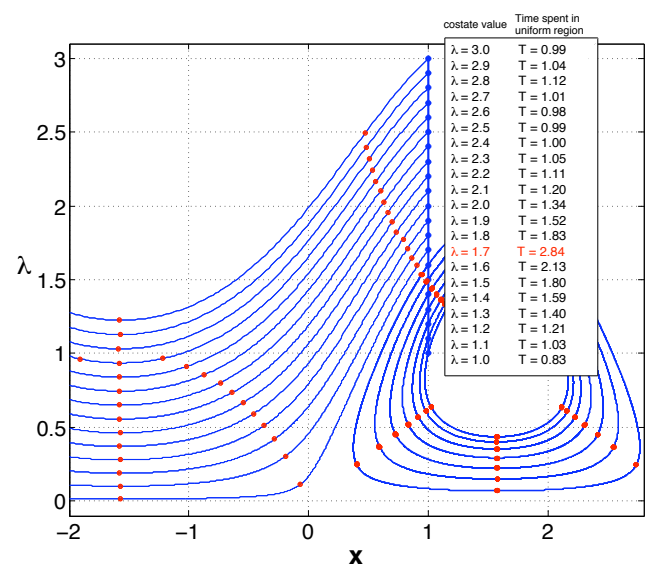

Fig. 4. Trajectories starting at $p(0)=(1, \lambda(0))$ for a grid of $\lambda(0)$ values. On each trajectory the zero $\rho_{h}$ points are noted. The maximum averaging time is noted for each value of $\lambda(0)$.
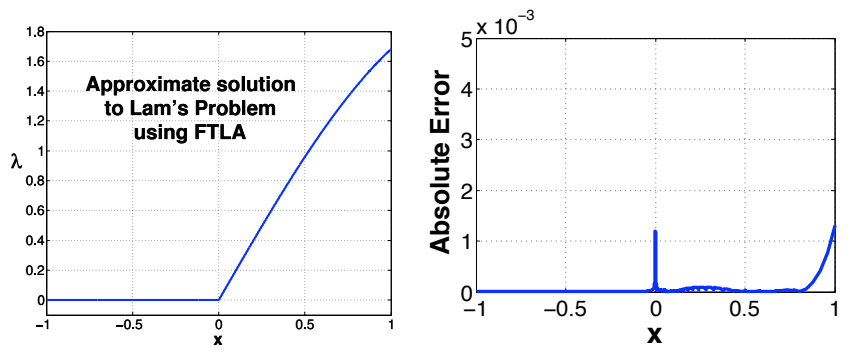

Fig. 5. Left: Approximation to Lam's problem using FTLA. The approximation to the stable invariant manifold is constructed with re-initializations for every $\mathrm{T}=0.1$. Right: The absolute error between the optimal solution from GESOP and the approximate solution constructed by FTLA vs $x$.

dichotomic basis. We have used kinematic eigenvalues to identify the regions where the instantaneous exponential behavior is uniform and select the appropriate averaging time for FTLA. Although we have only considered the completely hyper-sensitive case in this work, the method developed is general enough to be extended to partially hyper-sensitive problems.

\section{REFERENCES}

[1] www.gesop.de

[2] B.O. Anderson and P.K. Kokotovic, Optimal control problems over large time intervals, Automatica 23 (1972), 355 - 363.

[3] B.O. Anderson and P.V. Kokotovic, Optimal control problems over large time intervals, Automatica 23 (1987), no. 3, 355 - 363.

[4] U.M. Ascher, R.M. Mattheij, and R.D. Russell, Numerical solution of boundary value problems for ordinary differential eqautions, SIAM press, Philadelphia, 1995.

[5] L. Barreira and Y.B. Pesin, Lyapunov exponents and smooth ergodic theory, American Mathematical Society, Providence, USA, 2002.

[6] J.T. Betts, Survey of numerical methods for trajectory optimization, Journal of Guidance, Control, and Dynamics 21 (1998), no. 2, 193 207.

[7] R. Buizza and T.N. Palmer, The singular-vector structure of the atmospheric global circulation, J. Atmos. Sci. 52 (1995), no. 9, 1434 $-1459$.

[8] J.H. Chow, A class of singularly perturbed nonlinear, fixed end-point control problems, J. Opt. Theory Appl. 29 (1979), 231 - 251.

[9] R. Doerner, B. Hubinger, W. Martienssen, S. Grossmann, and S. Thomae, Stable manifolds and predictability of dynamical systems, Chaos, Solitons and Fractals 10 (1999), no. 11, 1759 - 1782.
[10] I. Goldhirsch, P.L. Sulem, and S.A. Orszag, Stability and Lyapunov stability of dynamical systems: A differential approach and a numerical method, Applied Dynamics and Computation 27 (1987), no. 3, $311-337$.

[11] G. Haller, Distinguished material surfaces and coherent structures in three-dimensional fluid flows, Physica D 149 (2001), 248 - 277.

[12] S.-H. Lam, Personal communication.

[13] K.D. Mease, S. Bharadwaj, and S. Iravanchy, Timescale analysis for nonlinear dynamical systems, J. Guidance Control and Dynamic 26 (2003), no. $1,318-330$.

[14] K.D. Mease, U. Topcu, and E. Aykutluğ, Characterizing two-timescale nonlinear dynamics using finite-time Lyapunov exponents and vectors, arXiv:0807.0239 [math.DS] (2008).

[15] A.V. Rao and K.D. Mease, Dichotomic basis approach to solving hyper-sensitive optimal control problems, Automatica 35 (1999), 3633-3642.

[16] __ Eigenvector approximate dichotomy basis method for solving hyper-sensitive optimal control problems, Optimal Control: Applications and Methods 21 (2000), 1-19.

[17] S. C. Shadden, F. Lekien, and J. E. Marsden, Definition and properties of Lagrangian coherent structures from finite-time Lyapunov exponents in two-dimensional aperiodic flows, Physica D 212 (2005), 271 - 304

[18] U. Topcu and K.D Mease, Using Lyapunov vectors and dichotomy to solve hyper-sensitive optimal control problems, Proceedings of the $45^{\text {th }}$ IEEE Conference on Decision and Control, 2006.

[19] J. A. Vastano and R. D. Moser, Short-time Lyapunov exponent analysis and the transition to chaos in taylor-couette low, J. Fluid Mechanics 233 (1991), 83 - 118.

[20] R.R. Wilde and P.V. Kokotovic, A dichotomy in linear control theory, IEEE Transactions on Automatic control 17 (1972), 382 - 383. 